Research Paper

\title{
The Neutrophil-Lymphocyte Ratio Predicts Recurrence of Cervical Intraepithelial Neoplasia
}

\author{
Sungwook Chun ${ }^{1 \#}$, Kyusik Shin ${ }^{2 \#, ~ K i ~ H y u n g ~ K i m ~}{ }^{3 * \bowtie}$, Heung Yeol Kim ${ }^{*} \bowtie$, Wankyu Eo ${ }^{5}$, Ji Young Lee ${ }^{6}$, \\ Jeong Namkung7, Sang Hoon Kwon ${ }^{8}$, Suk Bong Koh' ${ }^{9}$, Hong-Bae Kim ${ }^{10}$ \\ 1. Department of Obstetrics and Gynecology, College of Medicine, Inje University, Busan, Korea \\ 2. Department of Medicine, Pusan National University Graduate School, Busan, Korea \\ 3. Department of Obstetrics and Gynecology, Pusan National University School of Medicine, Biomedical Research Institute and Pusan Cancer Center, Pusan \\ National University Hospital \\ 4. Department of Obstetrics and Gynecology, College of Medicine, Kosin University, Busan, Korea \\ 5. Department of Internal Medicine, College of Medicine, Kyung Hee University, Seoul, Korea \\ 6. Department of Obstetrics and Gynecology, Research Institute of Medical Science, Konkuk University School of Medicine, Seoul, Korea \\ 7. Department of Obstetrics and Gynecology, The Catholic University of Korea College of Medicine, Seoul, Korea, Seoul, Korea \\ 8. Department of Obstetrics and Gynecology, Keimyung University, School of Medicine, Daegu, Korea \\ 9. Department of Obstetrics and Gynecology, Catholic University of Daegu, School of Medicine, Daegu, Korea \\ 10. Department of Obstetrics and Gynecology, Kangnam Sacred Heart Hospital, Hallym University Medical Center, Hallym University College of Medicine, \\ Seoul, Korea \\ \#Chun S and Shin K contributed equally to the study.
}

${ }^{*} \mathrm{Kim} \mathrm{HY}$ and Kim KH carry out equal roles as co-corresponding authors.

$\triangle$ Corresponding authors: Ki Hyung Kim, MD, PhD, Department of Obstetrics and Gynecology, Pusan National University School of Medicine, and Biomedical Research Institute and Pusan Cancer Center, Pusan National University Hospital, 179 Gudeok-ro, Seo-gu, Busan 49241, Republic of Korea. Tel: +82-51-240-7287; Fax: +82-51-248-2384. E-mail: ghkim@pusan.ac.kr and Heung Yeol Kim, MD, PhD, Department of Obstetrics and Gynecology, Kosin University College of Medicine, 34, Amnam-dong, Seo-gu, Busan 49267, Republic of Korea. Tel: +82-51-990-6226; Fax: +82-51-990-3300. E-mail: hykyale@yahoo.com.

(c) Ivyspring International Publisher. This is an open access article distributed under the terms of the Creative Commons Attribution (CC BY-NC) license (https://creativecommons.org/licenses/by-nc/4.0/). See http://ivyspring.com/terms for full terms and conditions.

Received: 2017.01.13; Accepted: 2017.04.25; Published: 2017.07.15

\begin{abstract}
OBJECTIVE: The purpose of the present study was to determine the prognostic significance of the neutrophil-lymphocyte ratio (NLR) in recurrence of cervical intraepithelial neoplasia (CIN).

METHODS: We evaluated the NLR as a prognostic marker in the entire cohort of 230 patients who had undergone surgical resection and were diagnosed with $\mathrm{CIN}$. Subjects were categorized into two different groups based on the NLR (NLR-high and NLR-low) using cutoff values determined by receiver operating characteristic (ROC) analysis. The primary research objective for this study was to validate the impact of the NLR on recurrence-free survival (RFS) in patients with CIN. The secondary objective was to evaluate the impact of other hematologic parameters on RFS in CIN patients.

RESULTS: Using the entire cohort, the most appropriate NLR cut-off value for CIN recurrence selected on the ROC curve was 2.1. The NLR-low and NLR-high groups included 167 (72.6\%) and 63 patients (27.4\%), respectively. According to Kaplan-Meier analysis, RFS rates during the entire follow-up period were considerably lower in the NLR-high group than in the NLR-low group ( $P=$ 0.0125). In multivariate survival analysis using Cox proportional hazard model, we identified the NLR, absolute eosinophil count (AEC), hemoglobin concentration, and mean corpuscular volume (MCV) as valuable prognostic factors that impact RFS.

CONCLUSIONS: The NLR is an independent prognosticator for RFS following surgical resection in CIN patients. We also found that the AEC, hemoglobin level, and MCV were strongly associated with RFS, as determined by multivariate analysis using a Cox model. These hematological parameters might provide additional prognostic value beyond that offered by standard clinicopathologic parameters.
\end{abstract}

Key words: Cervical Intraepithelial Neoplasia; Eosinophil; Lymphocyte; Neutrophil; Recurrence. 


\section{Introduction}

Cervical cancer is one of the most common causes of cancer death in women worldwide [1]. Cervical intraepithelial neoplasia (CIN) is preinvasive, preceding cervical cancer, and it is equivalent to the term cervical dysplasia. The concept of CIN was first introduced by Richart, who demonstrated that dysplasias have a high risk of progression to malignancy of the cervix [2]. The significant pathological features of CIN include immature cells, disorganization of the cells, abnormal nuclei, and increased mitotic activity [3]. Cervical lesions with mitoses and immature cells that are limited to the lower one-third of the epithelium are typically designated CIN 1, and involvement of the middle and upper one-third is diagnosed as CIN 2 and CIN 3, respectively [3]. Persistent infection with high-risk human papillomavirus (HR-HPV) is the single most important risk factor for the development of CIN [3-5]. HPV Type 16 is responsible for most $\mathrm{HPV}$-caused diseases such as invasive cancer, CIN 2 and CIN 3 [4].

Evidence suggests a variety of high-risk factors for CIN recurrence, including positive surgical margins [6, 7], histologic CIN grade [8], pretreatment HR-HPV infection or persistent infection with HR-HPV following surgical resection [7, 9-11], glandular involvement [7, 12-14], and immunosuppression $[13,14]$. Demographic factors such as age and menopausal status were also reported to be related to the recurrence of CIN to varying degrees [7, 11, 12]. $\mathrm{CIN}$ is a disease with a high potential for recurrence after surgery [7], and investigation of factors related to CIN recurrence is ongoing.

The causal relationship between inflammation and development of cancer has been well established [15-18]. In the tumor microenvironment, chronic inflammation contributes to promote tumor growth, tumor angiogenesis, invasion, metastasis, subversion of adaptive immune response, and reduced response to anticancer agents including hormones and chemotherapeutic agents [18, 19]. Chronic inflammation increases cancer risk, and although it is often undetectable, inflammation of any duration may have an essential role in carcinogenesis [18]. Recently, a number of studies investigated the most valuable measurement of the systemic inflammatory response and their potential use in clinical practice including cancer outcome prediction. Various easily measurable indices of blood that reflect the systemic inflammatory response have been recognized, including increased number of leukocytes, hypoalbuminemia, elevated C-reactive protein, and high cytokine levels [20-22].
Among these pretreatment blood parameters, the peripheral neutrophil-lymphocyte ratio (NLR), calculated as the absolute neutrophil count (ANC) divided by the absolute lymphocyte count (ALC), is regarded as a simple and effective marker of inflammation [23-25] that has been consistently reported to have an independent prognostic value in various tumors [21, 22, 26]. An increased NLR was associated with advanced stages of cervical neoplasia $[27,28]$, and patients with a higher histologic grade of CIN showed higher numbers of total leukocytes [29]. As far as we know, however, the prognostic value of the NLR for predicting CIN recurrence has not yet been reported. The aim of this study was to evaluate the prognostic significance of the NLR for recurrence in patients diagnosed with CIN of the uterus.

\section{Materials and Methods}

In this study, we retrospectively evaluated women diagnosed with CIN at different stages at two university hospitals. A total of 230 patients, aged 22 to 73 years, who underwent diagnostic excisional procedures for previous abnormal Papanicolaou smear results and were diagnosed with CIN were included in the study. Diagnostic excisional procedures such as conization or loop electrosurgical excision procedure (LEEP) with endocervical curettage (ECC) were performed by experienced gynecologists. Those who had received previous treatment for CIN, had evidence of infection including human immunodeficiency virus, or were using immunosuppressive drugs were excluded. This study was approved by the institutional review boards of both university hospitals for evaluation of patient data, and informed consent was waved considering the retrospective nature of data collection.

After a complete review of medical history, we collected information regarding patient demographics and clinicopathologic variables for analysis. The final cytological and pathological diagnosis of lesions followed the FIGO (International Federation of Gynecology and Obstetrics) classification, the histologic classification of which is based on the 2001 Bethesda System [30], and the classification of histological types was reviewed by a single expert pathologist for consistency. CIN recurrence was defined as histopathological CIN during follow-up. Hematology tests were performed in all participants prior to the operation as part of the routine work-up. Patients were excluded if their hematologic tests were not performed within two weeks before surgery. 


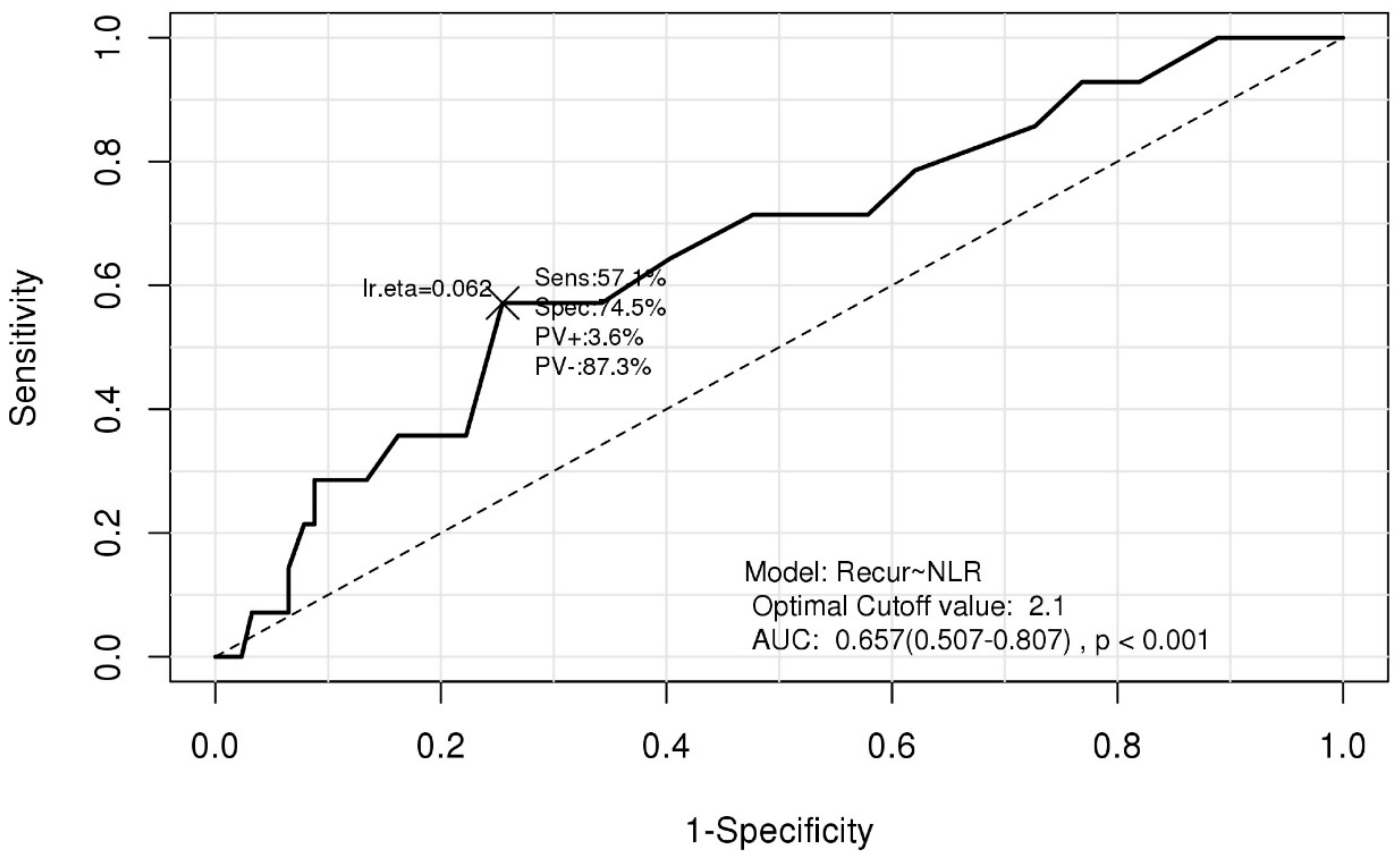

Figure 1. Receiver operating characteristic (ROC) curve used for determining the neutrophil-lymphocyte ratio cutoff value. AUC, area under the curve; NLR, neutrophil-lymphocyte ratio; Recur, recurrence; Sens, sensitivity; Spec, specificity; PV+, positive predictive value, PV-, negative predictive value.

Table 1. Clinicopathologic and laboratory parameters in patients with cervical intraepithelial neoplasia.

\begin{tabular}{ll}
\hline Characteristics & Mean \pm SD $(\mathbf{N}=\mathbf{2 3 0})$ \\
\hline Age & $45.4 \pm 10.6$ \\
Body mass index & $22.5 \pm 3.1$ \\
Treatment & \\
$\quad$ Conization & $213(92.6 \%)$ \\
LEEP with ECC & $17(7.4 \%)$ \\
Histology & $15(6.5 \%)$ \\
CIN1 & $43(18.7 \%)$ \\
CIN2 & $172(74.8 \%)$ \\
CIN3 & \\
Resection margin & $196(85.2 \%)$ \\
Negative & $34(14.8 \%)$ \\
Positive & $13.4 \pm 1.0$ \\
Hemoglobin & $90.8 \pm 6.2$ \\
MCV & $318.7 \pm 67.3$ \\
Platelet & $6161.2 \pm 1561.6$ \\
WBC & $3601.6 \pm 1308.4$ \\
ANC & $386.8 \pm 135.2$ \\
AMC & $120.9 \pm 100.3$ \\
AEC & $2045.1 \pm 560.7$ \\
ALC & $1.9 \pm 0.9$ \\
NLR & $166.8 \pm 57.3$ \\
PLR & $5.8 \pm 2.1$ \\
LMR & \\
HPV16 & $164(71.3 \%)$ \\
Negative & $66(28.7 \%)$ \\
Positive &
\end{tabular}

AEC, absolute eosinophil count; ALC, absolute lymphocyte count; AMC, absolute monocyte count; ANC, absolute neutrophil count; CIN, cervical intraepithelial neoplasia; HPV, human papilloma virus; LMR, lymphocyte-monocyte ratio; MCV, mean corpuscular volume; NLR, neutrophil-lymphocyte ratio; PLR,

platelet-lymphocyte ratio; WBC, white blood cell count.

Data from the entire cohort was used to choose optimal cutoff point of NLR for predicting recurrence based on receiver operating characteristic (ROC) curve analysis and determined that the optimal cutoff point of NLR for CIN recurrence was 2.1 (Fig. 1). We divided all patients into two groups depending on the cutoff value of NLR as follows: NLR-low (NLR $\leq 2.1)$ and NLR-high $(\mathrm{NLR}>2.1)$. Unpaired $t$-tests were applied for evaluation of continuous variables; independent-sample chi-squared tests were used for assessing categorical variables for comparison between the two groups.

In addition, we evaluated the impact of NLR inn recurrence-free survival (RFS). RFS was defined as the length of time between the date of excisional biopsy and the date of recurrence. Patients without documented disease recurrence were censored at the time of the last follow-up visit. RFS was analyzed using the Kaplan-Meier method; differences between survival curves were tested for statistical significance using log-rank tests. The univariate and multivariate Cox proportional hazards model was applied to identify the most significant independent prognosticator for RFS; variables with $P$-values of $<0.1$ on univariate analysis were included on multivariate analysis. All $P$-values are two-sided, and $P$-values of $<$ 0.05 were considered to be significant for statistical analyses. Data analysis was performed using IBM SPSS statistical software v. 18.0 (SPSS Inc., Chicago, IL, USA).

\section{Results}

The baseline characteristics of the patients are shown in Table 1. Two hundred thirteen patients (92.6\%) underwent conization, and 17 patients $(7.4 \%)$ 
underwent LEEP with ECC. In total, 15 (6.5\%), 43 $(18.7 \%)$, and 172 patients $(74.8 \%)$ were diagnosed with CIN I, CIN II, and CIN III, respectively. During the diagnostic excisional procedures, positive resection margins were reported in 34 patients $(14.8 \%)$, and $28.7 \%$ of women in our cohort tested positive for HPV 16.

Table 2. Comparison of the clinical and laboratory parameters between two groups of patients with cervical intraepithelial neoplasia divided according to neutrophil-lymphocyte ratio.

\begin{tabular}{|c|c|c|c|}
\hline & $\begin{array}{l}\text { NLR } \leq 2.1 \\
(\mathrm{~N}=167)\end{array}$ & $\begin{array}{l}\text { NLR>2.1 } \\
(N=63)\end{array}$ & $P$-value \\
\hline Age & $45.8 \pm 10.4$ & $44.3 \pm 11.1$ & $0.342^{a}$ \\
\hline Body mass index & $22.7 \pm 3.0$ & $22.0 \pm 3.2$ & $0.155^{a}$ \\
\hline Treatment & & & $1.000^{\mathrm{b}}$ \\
\hline Conization & $155(92.8 \%)$ & $58(92.1 \%)$ & \\
\hline LEEP with ECC & $12(7.2 \%)$ & $5(7.9 \%)$ & \\
\hline Histology & & & $0.995 \mathrm{~b}$ \\
\hline CIN1 & $11(6.6 \%)$ & $4(6.3 \%)$ & \\
\hline CIN2 & $31(18.6 \%)$ & $12(19.0 \%)$ & \\
\hline CIN3 & $125(74.9 \%)$ & $47(74.6 \%)$ & \\
\hline Resection margin & & & $0.938^{b}$ \\
\hline Negative & $143(85.6 \%)$ & $53(84.1 \%)$ & \\
\hline Positive & $24(14.4 \%)$ & $10(15.9 \%)$ & \\
\hline Hemoglobin & $13.4 \pm 1.0$ & $13.4 \pm 1.1$ & 0.983 a \\
\hline $\mathrm{MCV}$ & $90.8 \pm 6.6$ & $90.6 \pm 5.0$ & $0.804^{\mathrm{a}}$ \\
\hline Platelet & $315.7 \pm 69.7$ & $326.8 \pm 60.1$ & $0.267 \mathrm{a}$ \\
\hline WBC & $5829.8 \pm 1379.1$ & $7039.8 \pm 1683.4$ & $0.000^{a}$ \\
\hline ANC & $3130.4 \pm 933.4$ & $4850.5 \pm 1344.4$ & 0.000 a \\
\hline AMC & $383.1 \pm 124.8$ & $396.8 \pm 160.4$ & $0.540^{a}$ \\
\hline AEC & $130.8 \pm 104.4$ & $94.5 \pm 83.5$ & 0.007 a \\
\hline ALC & $2182.9 \pm 546.1$ & $1679.8 \pm 419.6$ & $0.000^{a}$ \\
\hline PLR & $152.6 \pm 50.7$ & $204.4 \pm 57.1$ & $0.000 \mathrm{a}$ \\
\hline LMR & $6.2 \pm 2.2$ & $4.7 \pm 1.5$ & $0.000 \mathrm{a}$ \\
\hline HPV16 & & & $0.850^{\mathrm{b}}$ \\
\hline Negative & $118(70.7 \%)$ & $46(73.0 \%)$ & \\
\hline Positive & $49(29.3 \%)$ & $17(27.0 \%)$ & \\
\hline \multicolumn{4}{|c|}{ a $P$-valuesbyunpaired $t$-test. } \\
\hline \multicolumn{4}{|c|}{$\begin{array}{l}\text { AEC, absolute eosinophil count; ALC, absolute lymphocyte count; AMC, absolute } \\
\text { monocyte count; ANC, absolute neutrophil count; CIN, cervical intraepithelial } \\
\text { neoplasia; HPV, human papilloma virus; LMR, lymphocyte-monocyte ratio; MCV, } \\
\text { mean corpuscular volume; NLR, neutrophil-lymphocyte ratio; PLR, } \\
\text { platelet-lymphocyte ratio; WBC, white blood cell count. }\end{array}$} \\
\hline
\end{tabular}

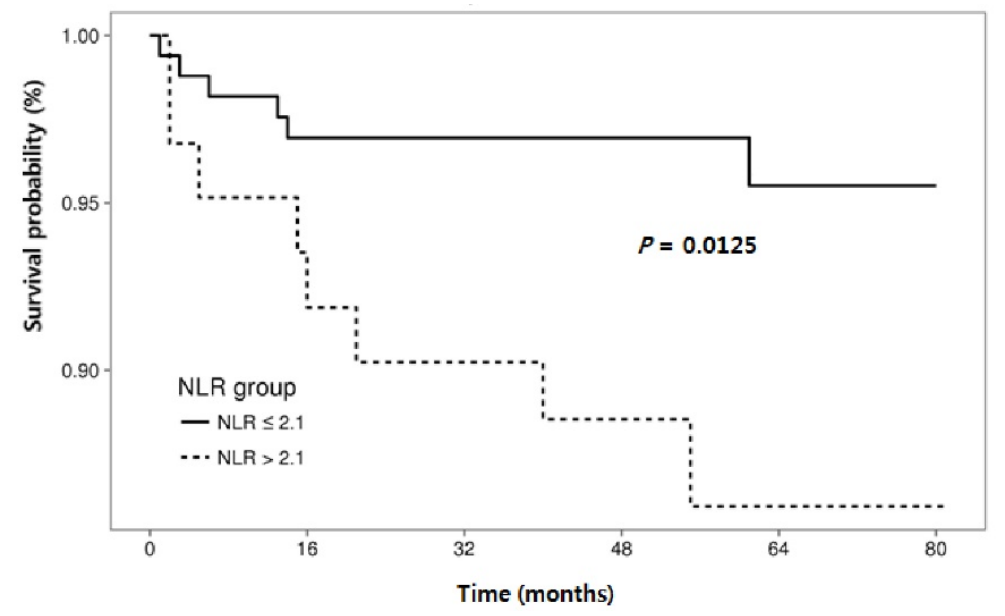

Figure 2. Cumulative recurrence-free survival according to neutrophillymphocyte ratio.
Table 2 shows the comparison of baseline characteristics between the two groups categorized according to the NLR cut-off values: NLR-low $(\mathrm{n}=$ $167,72.6 \%)$ and NLR-high group ( $\mathrm{n}=62,27.4 \%)$. Significant mean differences between the two groups were found in the following continuous variables: white blood cell count (WBC), absolute neutrophil count (ANC), absolute eosinophil count (AEC), absolute lymphocyte count (ALC), platelet-lymphocyte ratio (PLR), and lymphocyte-monocyte ratio (LMR). However there was no significant difference between the two groups for the categorical variables such as type of surgical treatment, histologic grade, rate of positive resection margins, or HPV 16 infection rate.

The RFS rates during the entire follow-up period were significantly lower in the NLR-high group compared to the NLR-low group $(P=0.0125)$ (Fig. 2$)$. Regarding other hematological parameters (Fig. 3), the RFS rates were significantly higher in those groups with low-ANC $(P=0.0225)$, high-ALC $(P=$ $0.0342)$, low-AEC $(P=0.0077)$, and high-hemoglobin level $(P=0.0167)$. Patients with higher mean corpuscular volume (MCV) showed a trend toward higher RFS rates, but the difference did not make a significant significance $(P=0.0674)$.

The outcomes of univariate analysis for RFS identified significant variables, which are shown in Table 3. Age, ANC, AEC, ALC, hemoglobin level, and NLR were variables with significant differences on univariate analysis for RFS. Using the multivariate Cox proportional hazards model, we identified NLR (hazard ratio [HR], 7.66; 95\% confidence interval [CI], 2.34-25.10; $P=0.001)$, AEC (HR, 6.91; 95\% CI, 1.82-26.24; $P=0.005)$, hemoglobin level (HR, $0.21 ; 95 \%$ CI, 0.07-0.65; $P=0.007)$, and MCV (HR, $0.27 ; 95 \% \mathrm{CI}$, $0.08-0.92 ; P=0.037$ ) as the strongest prognostic factors (Table 3).

\section{Discussion}

CIN or cervical dysplasia has a relatively high potential for recurrence after surgery [7]. Most cases of mild cervical dysplasia regress spontaneously to normal [31], whereas high-grade CIN is regarded as a lesion that can progress to invasive carcinoma if left untreated [3]. However, a variety of factors, except the histologic grade of CIN, have also been shown to be related to the recurrence of cervical dysplasia [6-14]; thus, further investigation of the parameters related to CIN recurrence is needed. Leukocytosis and neutrophilia are one of the most frequently encountered alterations in cancer 


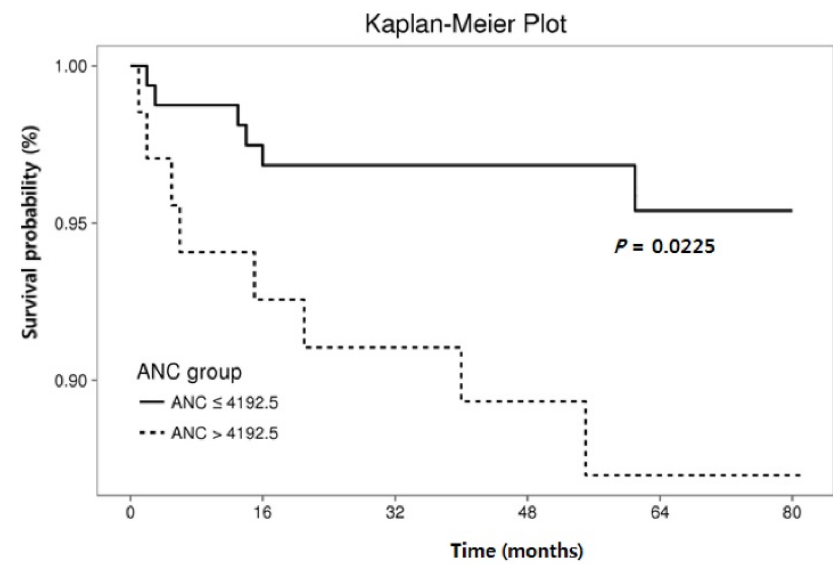

patients [22, 27], and these findings correlate significantly with advanced disease and, consequently, with prognosis $[22,27,28]$. The NLR has been regarded as a non-invasive and cost-effective marker that reflects systemic inflammatory conditions [22, 26, 32], and many studies have reported its prognostic value in diverse cancers [21, 22, 33-35]. The current study is, as far as we known, the first report on the association between NLR and CIN recurrence, and we showed that NLR is an independent prognostic factor for disease recurrence after surgical resection in CIN patients. Since Virchow first described the relationship between chronic inflammation and cancer [36], the association has become more widely recognized $[15,16,19]$. Continuous cell proliferation in a millieu rich in inflammatory cells including neutrophils and macrophpages, cell growth factors, angiogenic and lymphangiogenic growth factors, stroma, and DNA-damage-promoting agents promotes the cancer development [15]. Following tumorigenesis, cancer cells produce different arrays of cytokines and chemokines that are mitogenic and/or chemoattractant for various cells, including granulocytes, mast cells, monocytes/macrophages, fibroblasts, and endothelial cells [15, 37]. Additionally, activation of fibroblasts and infiltrating inflammatory cells secrete proteolytic enzymes, cytokines, and chemokines, which are mitogenic for cancer cells, as well as endothelial cells involved in angiogenesis; these factors augment growth of cancer cell and enable metastatic spread [15]. Several types of infection are well known to potentiate cancer development and dissemination; for example, Helicobacter pylori infection is associated with gastric cancer and mucosa-associated lymphoid tissue lymphoma, Epstein-Barr virus infection potentiate development of various kinds of malignant lymphoma, and hepatitis B or C virus infection increases the risk of hepatoma $[15,18]$.
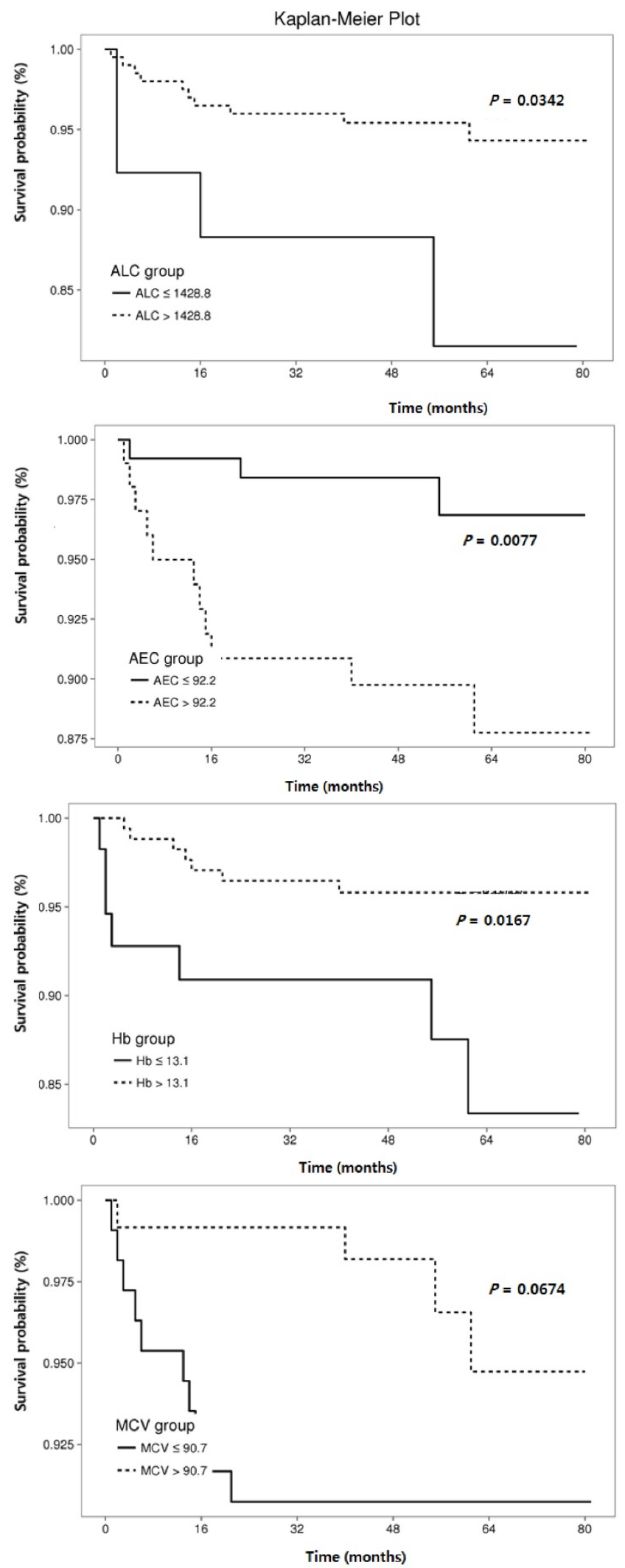

Figure 3. Cumulative recurrence-free survival according to hematologic parameters other than neutrophil-lymphocyte ratio. 
Table 3. Relationships between clinical and laboratory parameters and recurrence-free survival in patients with cervical intraepithelial neoplasia.

\begin{tabular}{|c|c|c|c|c|}
\hline \multirow[b]{2}{*}{ Variable } & \multicolumn{2}{|l|}{ Univariate } & \multicolumn{2}{|l|}{ Multivariate } \\
\hline & HR $(95 \% \mathrm{CI})$ & $\begin{array}{l}\text { P-val } \\
\text { ue }\end{array}$ & HR $(95 \%$ CI) & $\begin{array}{l}\text { P-val } \\
\text { ue }\end{array}$ \\
\hline Age (years) $(\leq 40$ vs. $>40)$ & $\begin{array}{l}4.62 \\
(1.03-20.65\end{array}$ & 0.045 & & \\
\hline Body mass index ( $\leq 23$ vs. $>23$ ) & $\begin{array}{l}2.62 \\
(0.91-7.55)\end{array}$ & 0.075 & & \\
\hline $\begin{array}{l}\text { ANC (per } \mu \mathrm{L})(\leq 4192.5 \mathrm{vs} \text {. } \\
>4192.5)\end{array}$ & $\begin{array}{l}3.22 \\
(1.12-9.27)\end{array}$ & 0.031 & & \\
\hline $\mathrm{AEC}($ per $\mu \mathrm{L})(\leq 92.2$ vs. $>92.2)$ & $\begin{array}{l}4.80(1.34 \\
17.20)\end{array}$ & 0.016 & $\begin{array}{l}6.91 \\
(1.82-26.24)\end{array}$ & 0.005 \\
\hline $\begin{array}{l}\text { ALC (per } \mu \mathrm{L})(\leq 1428.8 \text { vs. } \\
>1428.8)\end{array}$ & $\begin{array}{l}0.31 \\
(0.10-0.98)\end{array}$ & 0.045 & & \\
\hline $\begin{array}{l}\text { Hemoglobin }(\mathrm{g} / \mathrm{dL})(\leq 13.1 \text { vs. } \\
>13.1)\end{array}$ & $\begin{array}{l}0.30 \\
(0.11-0.86\end{array}$ & 0.024 & $\begin{array}{l}0.21 \\
(0.07-0.65)\end{array}$ & 0.007 \\
\hline MCV (fL) ( $\leq 90.7$ vs. > 90.7) & $\begin{array}{l}0.35 \\
(0.11-1.13)\end{array}$ & 0.079 & $\begin{array}{l}0.27 \\
(0.08-0.92)\end{array}$ & 0.037 \\
\hline $\operatorname{NLR}(\leq 2.1$ vs. $>2.1)$ & $\begin{array}{l}3.54(1.23, \\
10.20)\end{array}$ & 0.019 & $\begin{array}{l}7.66 \\
(2.34-25.10)\end{array}$ & 0.001 \\
\hline
\end{tabular}

Hazard ratios were obtained using Cox's proportional hazard model.

AEC, absolute eosinophil count; ALC, absolute lymphocyte count; ANC, absolute neutrophil count; $\mathrm{MCV}$, mean corpuscular volume; NLR, neutrophil-lymphocyte ratio.

It is also well established that the systemic inflammatory response is associated with alterations in circulating WBCs like neutrophilia and lymphocytopenia. In the clinic, hematology tests including WBC level are routinely carried out prior to cancer treatment; hence, the tests may help estimate the severity of the inflammatory response in cancer patients. [18, 21, 23]. Neutrophilia may be related to an environment favorable for development and progression of tumors; conversely, lymphocytes exert antitumor effects by inhibiting cell proliferation and migration; hence, a higher NLR is indicative of advanced or aggressive tumor behavior and negative treatment outcomes [21, 26].

Uterine cervical cancer is one of the most common malignancies and leading cause of cancer death among women worldwide [1], and it is preceded by a pre-invasive step in which defective, impaired neutrophil migration could be an early event in tumor development [28]. Tavares-Murta BM et al. [27] conducted a study in a total of 315 patients to evaluate the peripheral blood WBC counts in uterine cervical cancer and CIN. Compared with CIN and early-stage cervical cancer, leukocytosis, neutrophilia, lymphopenia, and a NLR $\geq 5$ were more frequently observed in advanced stage cervical cancer patients; moreover, neutrophilia was more frequent even in the early stage cervical cancer, when comparing with the CIN group.

Several studies demonstrated the prognostic value of the pretreatment NLR in cervical cancer [26, 32, 38]. In 235 patients who underwent definitive concurrent chemoradiation therapy (CCRT) for cervical cancer, the pretreatment NLR was associated with tumor size, lymph node metastasis, and response to therapy, and pretreatment NLR was found to independently predict survival outcomes [26]. Other studies also showed that a pretreatment NLR was a significant prognosticator for survival in patients with cervical cancer [32, 38]. Inflammatory components are critical in the development and progression of cervical cancer, like in other cancers, and the NLR is a feasible biomarker to predict patient outcomes and guide adjuvant therapy in cervical cancer [26].

Eosinophils are considered to be one of the first recruited effectors of the acute inflammatory response [15]. In the present study, AEC was significantly associated with CIN recurrence on multivariate analysis. In previous studies, the blood AEC level has been reported to be related to the stage of the cervical and rectal cancers. In a study by Dalai et al, the blood AEC level in patients with stages III and IV cervical cancer was higher when compared with that of stages I and II disease. In addition, AEC level before treatment was useful in predicting radiation response $[39,40]$, a finding that is partially consistent with our results that the high-AEC group had lower RFS. However, the clinical significance of AEC in CIN is not certain; as the value of AEC as a predictor for recurrence of CIN has not been reported, the result of current study needs validation in subsequent studies.

In this study, pretreatment hemoglobin level was identified as valuable prognostic factors for RFS. In previous studies on cervical cancer, low pretreatment hemoglobin level has been associated with increased risk of cervical cancer recurrence [41-43], and these findings are partially consistent with our own.

In current study, pretreatment $\mathrm{MCV}$ value was identified as valuable prognostic factors for RFS. As far as we know, the clinical significance of MCV in CIN has not been reported, and our results need validation in subsequent studies.

This study has some limitations; it was a retrospective observational study with a relatively small sample size. In addition, we were unable to adjust for some critical confounders such as systemic infection or inflammation; like other non-specific markers of inflammation, the NLR may have been affected by the presence of other systemic diseases [44]. Finally, although we evaluated the role of NLR considering other inflammatory indices like PLR and LMR, we could not evaluate the Glasgow Prognostic Score (GPS) [19] due to a lot of missing values in the blood chemistry data. If we had investigated the prognostic value of the NLR in combination with diverse prognostic markers of systemic inflammation including GPS, the clinical value NLR for predicting CIN recurrence could have been more precisely 
determined. Further research is necessary in this regard.

In conclusion, this study was the first attempt to evaluate the prognostic value of the NLR for predicting CIN recurrence, and we found that NLR is an independent prognostic factor for RFS after surgical excision of CIN. Additionally, we found that the AEC, hemoglobin level, and MCV were strongly associated with RFS, as determined by multivariate analysis using a Cox model. These hematological parameters might provide additional prognostic value beyond that offered by standard clinicopathologic parameters. Further large-scale trials are needed to clarify the preliminary findings of the present study.

\section{Acknowledgements}

This study was supported by a grant from the National R\&D Program for Cancer Control, Ministry for Health, Welfare, and Family Affairs, Republic of Korea (0920050).

\section{Competing Interests}

The authors have declared that no competing interests exist.

\section{References}

1. [Internet] Global cancer facts and figures. http://www.cancer.org/ downloads/STT/Global_Cancer_Facts_andFigures_2007_rev.pdf.

2. Richart RM. Natural history of cervical intraepithelial neoplasia. ClinObstet Gynecol. 1968; 10: 748.

3. Garcia F, Hatch KD, Berek JS. Intraepithelial Disease of the Cervix, Vagina, and Vulva. In: BerekJS, ed. Berek \& Novak's Gynecology, 15th ed. Philadelphia: Lippincott Williams \& Wilkins; 2012: 574-618.

4. Lorincz AT, Reid R, Jenson AB, et al. Human papilloma virus infection of the cervix: relative risk associations of 15 common anogenital types. Obstet Gynecol. 1992; 79: 328-37.

5. Lorincz AT, Richart RM. Human papillomavirus DNA testing as an adjunct to cytology in cervical screening programs. Arch Pathol Lab Med. 2003; 127: 959-68.

6. Serati M, Siesto G, Carollo S, et al. Risk factors for cervical intraepithelial neoplasia recurrence after conization: a 10-year study. Eur J ObstetGynecolReprod Biol. 2012; 165: 86-90.

7. Jin $\mathrm{J}$, Li L, Zhang F. META-ANALYSIS OF HIGH RISK FACTORS OF RESIDUE OR RELAPSE OF CERVICAL INTRAEPITHELIAL NEOPLASIA AFTER CONIZATION. J BiolRegulHomeost Agents.2015;29:451-8.

8. Alonso I, Torne' A, Puig-Tintore' LM, et al. High-risk cervical epithelial neoplasia grade 1 treated by loop electrosurgical excision procedure: follow-up and value of human papillomavirus testing. Am J Obstet Gynecol. 2007; 197: 359.e1 -.e6.

9. Verguts J, Bronselaer B, Donders G, et al. Prediction of recurrence after treatment for high-grade cervical intraepithelial neoplasia: the role of human papillomavirus testing and age at conisation. BJOG.2006;10:1303-7.

10. Yokoyama M, Iwasaka T, Nagata C, et al. Prognostic factors associated with the clinical outcome of cervical intraepithelial neoplasia: a cohort study in Japan. Cancer Lett.2003;192:171-9.

11. Prato B, Ghelardi A, Gadducci A, et al. Correlation of recurrence rates and times with posttreatment human papillomavirus status in patients treated with loop electrosurgical excision procedure conization for cervical squamous intraepithelial lesions. Int J Gynecol Cancer.2008;18:90-4.

12. Paraskevaidis E, Lolis ED, Koliopoulos G, et al. Cervical intraepithelial neoplasia outcomes after loop excision with clear margins. Obstet Gynecol. 2000; 95: 828-31.

13. Lima MI, Tafuri A, Arau jo AC, Lima LM, Melo VH. Cervical intraepithelial neoplasia recurrence after conization in HIV-positive and HIV-negative women. Int J Gynecol Obstet. 2009; 104: 100-4.

14. Teixeira Lodi C, Michelin MA, Lima MI, et al. Factors associated with recurrence of cervical intraepithelial neoplasia after conization in HIV-infected and noninfected women. Arch Gynecol Obstet. 2011; 284: 191-7.

15. Coussens LM, Werb Z. Inflammation and cancer. Nature. 2002; 420: 860-7.
16. Babu SN, Chetal G, Kumar S. Macrophage Migration Inhibitory Factor: a Potential Marker for Cancer. Asian Pac J Cancer Prev. 2012; 13: 1737-44.

17. Mantovani A, Allavena P, Sica A, Balkwill F. Cancer-related inflammation. Nature. 2008; 454: 436-44

18. Grivennikov SI, Greten FR, Karin M. Immunity, inflammation, and cancer. Cell. 2010; 140: 883-99.

19. Mantovani A, Garlanda C, Allavena P. Molecular pathways and targets in cancer-related inflammation. Ann Med. 2010; 42: 161-70.

20. McMillan DC. The systemic inflammation-based Glasgow Prognostic Score: a decade of experience in patients with cancer. Cancer Treat Rev. 2013; 39: 534-40.

21. Guthrie GJ, Charles KA, Roxburgh CS, et al. The systemic inflammation-based neutrophil-lymphocyte ratio: experience in patients with cancer. Crit Rev OncolHematol. 2013; 88: 218-30.

22. Templeton AJ, McNamara MG, Šeruga B, et al. Prognostic role of neutrophil-to-lymphocyte ratio in solid tumors: a systematic review and meta-analysis. J Natl Cancer Inst. 2014; 106: dju124.

23. Zahorec R. Ratio of neutrophil to lymphocyte counts - rapid and simple parameter of systemic inflammation and stress in critically ill. BratislavskeLekarskeListy. 2001; 102: 5-14.

24. Acmaz G, Aksoy H, Unal D, et al. Are Neutrophil/Lymphocyte and Platelet/Lymphocyte Ratios Associated with Endometrial Precancerous and Cancerous Lesions in Patients with Abnormal Uterine Bleeding? Asian Pac J Cancer Prev. 2014; 15: 1689-92.

25. Wang GY, Yang Y, Li H, et al. A scoring model based on neutrophil to lymphocyte ratio predicts recurrence of HBV-associated hepatocellular carcinoma after liver transplantation. PLoS One. 2011; 6: e25295.

26. Onal C, Guler OC, Yildirim BA. Prognostic Use of Pretreatment Hematologic Parameters in Patients Receiving Definitive Chemoradiotherapy for Cervical Cancer. IntJ Gynecol Cancer. 2016; 26: 1169-75.

27. Tavares-Murta BM, Mendonça MA, Duarte NL, et al. Systemic leukocyte alterations are associated with invasive uterine cervical cancer. Int J Gynecol Cancer. 2010; 20: 1154-9.

28. Fernandes PC Jr, Garcia CB, Micheli DC, et al. Circulating neutrophils may play a role in the host response in cervical cancer. Int J Gynecol Cancer. 2007; 17: $1068-74$

29. Bais AG, Beckmann I, Lindemans J, et al. A shift to a peripheral Th2-type cytokine pattern during the carcinogenesis of cervical cancer becomes manifest in CIN III lesions. J ClinPathol. 2005; 58: 1096-100.

30. Solomon D, Davey D, Kurman R, et al. The 2001 Bethesda System: terminology for reporting results of cervical cytology. JAMA. 2002; 287: 2114-9.

31. Nasiell K, Roger V, Nasiell M. Behavior of mild cervical dysplasia during long-term follow-up. Obstet Gynecol. 1986; 67: 665-9.

32. Mizunuma M, Yokoyama $\mathrm{Y}$, Futagami $\mathrm{M}$, et al. The pretreatment neutrophil-to-lymphocyte ratio predicts therapeutic response to radiation therapy and concurrent chemoradiation therapy in uterine cervical cancer. Int J ClinOncol. 2015; 20: 989-96.

33. Sarraf KM, Belcher E, Raevsky E, et al. Neutrophil/lymphocyte ratio and its association with survival after complete resection in non-small cell lung cancer. J ThoracCardiovasc Surg. 2009; 137: 425-8.

34. Tsai PL, Su WJ, Leung WH, Lai CT, Liu CK. Neutrophil-lymphocyte ratio and CEA level as prognostic and predictive factors in colorectal cancer: A systematic review and meta-analysis. J Cancer Res Ther. 2016; 12: 582-9.

35. Pichler M, Hutterer GC, Stoeckigt C, et al. Validation of the pre-treatment neutrophil-lymphocyte ratio as a prognostic factor in a large European cohort of renal cell carcinoma patients. Br J Cancer. 2013; 108: 901-907.

36. Balkwill F, Mantovani A. Inflammation and cancer: back to Virchow? Lancet. 2001; 357: 539-45.

37. Mantovani A, Bottazzi B, Colotta F, Sozzani S, Ruco L. The origin and function of tumor-associated macrophages. Immunol Today. 1992; 13: 265-70.

38. Lee YY, Choi CH, Kim HJ, et al. Pretreatment neutrophil: lymphocyte ratio as a prognostic factor in cervical carcinoma. Anticancer Res. 2012; 32: 1555-61.

39. Dalal BI, Das KC, Dutta TK, Malakar K. Local and Systemic Eosinophilia in Patients with Carcinoma of the Uterine Cervix Undergoing Radiation Therapy: Correlation with Radiation Response. ClinOncol (R CollRadiol). 1992; 4: 18-21.

40. Fisher ER, Paik SM, Rockette H, et al.Prognostic significance of eosinophils and mast cells in rectal cancer: findings from the National Surgical Adjuvant Breast and Bowel Project (protocol R-01). Hum Pathol. 1989; 20: 159-63.

41. Liu SC, Huang EY, Hu CF, et al. Pretreatment Factors Associated with Recurrence for Patients with Cervical Cancer International Federation of Gynecology and Obstetrics Stage IB1 Disease. GynecolObstet Invest. 2016; 81: 339-45.

42. Lim A, Sia S. Outcomes of chemoradiotherapy in cervical cancer- the Western Australian experience. Int J RadiatOncolBiol Phys. 2012; 82: 1431-8.

43. Barkati M, Fortin I, Mileshkin L, et al. Hemoglobin level in cervical cancer: a surrogate for an infiltrative phenotype. Int J Gynecol Cancer. 2013; 23: 724-9.

44. EoWK, Kwon S, Koh SB, et al. The Lymphocyte-Monocyte Ratio Predicts Patient Survival and Aggressiveness of Endometrial Cancer. J Cancer.2016;7:538-45. 